\title{
Chemical and electrical properties of Yb-doped strontium cerates in coal combustion atmospheres
}

\author{
J. Luyten ', F. De Schutter \\ Studiecentrum voor Kernenergie, SCK/CEN, Boeretang 200, $2400 \mathrm{Mol}$, Belgium \\ J. Schram and J. Schoonman \\ Laboratorium voor Anorganische Chemie, Technische Universiteit Delft, Julianalaan 136, 2628 BL Delft. The Netherlands
}

Received 16 September 1990; accepted for publication 28 January 1991

\begin{abstract}
This paper describes the development of $\mathrm{Yb}$-doped strontium cerates for use in a hydrogen sensor in coal gasification atmospheres. The first results of a characterization study of the microstructural as well as mechanical properties are presented. A comparison between virgin samples and samples exposed to a synthetic coal gasification atmosphere indicated no deleterious effect by this environment. Also the preliminary results with respect to an electrical characterization confirm this observation and are in agreement with the results of previous investigations.
\end{abstract}

\section{Introduction}

In the field of combined cycle power generation systems several advanced coal technologies are considered. However, optimisation of the electricity production with combined cycles requires knowledge of the gas composition inside the reactor.

In the framework of the European efforts on nonnuclear energy production (JOULE), a joint program between the SCK, Mol and the TU, Delft was started to develop a hydrogen sensor for these aggressive atmospheres.

In a first approach a sensor capable of operating at temperatures of about $900^{\circ} \mathrm{C}$ is envisaged. For this purpose, a galvanic cell using doped cerates as ion. conducting element was selected. In this stage, the behaviour of this material in the aggressive atmosphere is of prior importance. Therefore a program was started to test compatibility of the material in a simulated coal gas atmosphere.

In order to synthesize strontium cerate in the form of pellets and bars, the procedure as described by Iwahara et al. [1] was followed. To show the influence of dopant on the defect structure, three differ-

\footnotetext{
I Author to whom all correspondence should be addressed.
}

ent $\mathrm{Yb}$ containing compositions are manufactured: $\mathrm{SrCeO}_{3}, \mathrm{SrCe}_{0.95} \mathrm{Yb}_{0.05} \mathrm{O}_{3-\alpha}$ and $\mathrm{SrCe}_{0.9} \mathrm{Yb}_{0.1} \mathrm{O}_{3-\alpha}$. After synthesis, the ceramics were characterised in depth using several analysing techniques such as $\mathrm{Hg}$ porosimetry, gas permeability, SEM, TEM, XRD, and ceramography. Finally, also the mechanical properties were measured by performing four point bending tests on the square bars.

In order to measure the electrical properties, an impedance spectroscopy analysis (IS) was performed using a SOLARTRON 1255 frequency response analyser in combination with a SOLARTRON 1286 electrochemical interface at temperatures varying from 373 to $1173 \mathrm{~K}$.

\section{Experimental}

After the characterisation described above, specimens were exposed at $800^{\circ} \mathrm{C}$ to a simulated coal gas atmosphere in order to observe the high temperature stability of the component. In order to simulate a coal gas atmosphere, a gas mixture consisting of argon, $\mathrm{H}_{2}$ ( $\left.5 \mathrm{vol} \%\right), \mathrm{CO}(0.45 \mathrm{vol} \%), \mathrm{H}_{2} \mathrm{O}(0.08 \mathrm{vol} \%)$ and $\mathrm{H}_{2} \mathrm{~S}$. $(0.0033 \mathrm{vol} \%)$ at atmospheric pressure was used. A calculation of the thermodynamic parame- 
ters at $1073 \mathrm{~K}$ yields the following activity values:

$P_{\mathrm{O}_{2}}$ (bar) $=1.0 \times 10^{-22}$,

$P_{\mathrm{S}_{2}}($ bar $)=0.9 \times 10^{-10}$,

$a_{\mathrm{C}}=0.04$.

During exposure, specimens were placed in a closed quartz tube of $10 \mathrm{~cm}$ diameter mounted in a tube furnace. As the furnace is equipped with three independent heating coils, a temperature gradient of less than $5 \mathrm{~K}$ over a length of $10 \mathrm{~cm}$ could be obtained. Furthermore, the system is equipped with $\mathrm{H}_{2} \mathrm{~S}$-, $\mathrm{CO}$ - and $\mathrm{H}_{2}$-sensors for gas control. A stabilised $\mathrm{H}_{2} \mathrm{O}$ content was obtained by leading the gas through a mixture of $90 \%$ oxalic acid dihydrate and $10 \%$ of anhydrous acid kept in a thermostat at 280 $\mathrm{K}$. The amount of $\mathrm{H}_{2} \mathrm{O}$ was continuously monitored by a hygrometer (General Eastern 1100 DP). After an exposure of $100 \mathrm{~h}$, the same material analysis as mentioned above was performed including weight change measurements.

\section{Results}

\subsection{Material properties before exposure}

Using $\mathrm{Hg}$ porosimetry, a relative density of about $88 \%$ td was measured for the Yb-doped cerates and about $82 \%$ td for the undoped cerates. In the doped samples, about $12 \%$ open porosities were measured ( $18 \%$ in the undoped sample).

Moreover, as the Yb content increases, the specimens show an increasing density. These observations are confirmed by the microscopic investigation of the $\mathrm{SrCeO}_{3}$-based ceramics. Also a green surface region at the circumference of the specimen was observed.

With respect to the microstructure, SEM analysis of a fractured sample revealed a fracture of a full intercrystalline type with a mean grain size of about 8 $\mu \mathrm{m}$. This result and the presence of pores was confirmed by a TEM-observation of the microstructure. In addition, as shown in fig. 1, an amorphous phase between the grains was detected. However, it should be mentioned that the amorphous structures did not influence the overall crystal structure of the ceramics, as microstructure $\mathrm{XRD}$-analysis revealed the

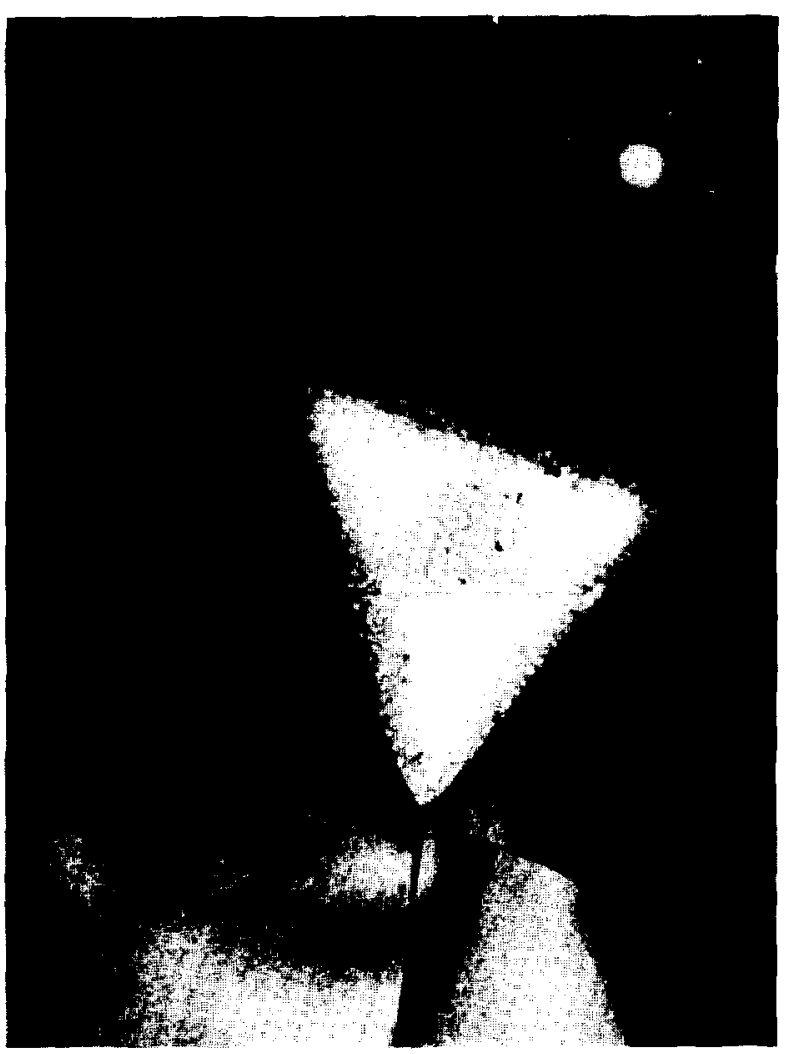

Fig. 1. TEM examination of a $\mathrm{SrCe}_{0.95} \mathrm{Yb}_{0.05} \mathrm{O}_{3-\alpha}$ sample showing the amorphous phase observed at triple points of the grains. Insert: Diffraction pattern.

Table 1

Residual strength of $\mathrm{SrCe}_{0.95} \mathrm{Yb}_{0.05} \mathrm{O}_{3-\alpha}$ after $100 \mathrm{~h}$ exposure to the coal gas atmosphere and after quenching from $900^{\circ} \mathrm{C}$.

\begin{tabular}{ll}
\hline 4-point bend strength (MPa) \\
\hline uncorroded & $36.3 \pm 2.4$ \\
after one time quenching & $28.6 \pm 13.0$ \\
after five times quenching & $\approx 0$ \\
after 100 h exposure & $44.5 \pm 8.2$ \\
\hline
\end{tabular}

expected $\mathrm{SrCeO}_{3}$ structure for all three compositions.

The mechanical properties of specimens before and after exposure and specimens which are one and five times quenched from $900^{\circ} \mathrm{C}$ are summarised in table 1 .

The results of four-point bending tests demonstrate the very low mechanical strength of the specimens. The quenching tests of the specimens, which 
were originally performed to provide an indication of the thermal shock resistance, reveal a very deleterious effect. After five times quenching the mechanical strength is nearly reduced to zero.

\subsection{Material properties after exposure}

After the exposure to the simulated coal-gas atmosphere the specimens remained intact but a change in colour from green (and yellow in the interior) to black could be detected. However, a cross-section of a specimen clearly showed that this colour change did not extend into the inner part of the material, and only the green surface layer mentioned above is transformed into a black one.

The weight differences before and after exposure are very low and dependent on the density of the specimens. There is a small material loss, but the less dense specimens have absorbed some components of the coal-gas atmosphere during quenching. XRDanalysis of the specimens after exposure reveal the existence of $\mathrm{SrS}$ and $\mathrm{CeO}_{2}$ in addition to $\mathrm{SrCeO}_{3}$. The reaction products $\mathrm{SrS}$ and $\mathrm{CeO}_{2}$ could not be detected in the interior of the sample.

Finally it should be mentioned that the $100 \mathrm{~h}$ ex- posure to the coal-gas atmosphere resulted only in a slight increase of the original strength, probably caused by the surface transformation.

\subsection{Impedance measurements}

Up to now, IS was performed in air on the $\mathrm{SrCe}_{0.95} \mathrm{Yb}_{0.05} \mathrm{O}_{3-\alpha}$ specimens only before and after exposure. Impedance spectra were recorded in the temperature range 425 to $930 \mathrm{~K}$, with intervals of $\pm 100 \mathrm{~K}$. At the lowest temperature, three semicircles, indicating the existance of grain boundary effects can be detected. However, due to experimental limitations, only part of these circles could be reproduced at the higher temperatures. IS performed after the exposure, yield almost the same spectra at all temperatures. This is a first indication that the exposure does not alter the electrical properties.

\section{Discussion}

The detailed evaluation of the microstructural as well as the mechanical properties of the specimens synthesised according to the procedure of Iwahara et

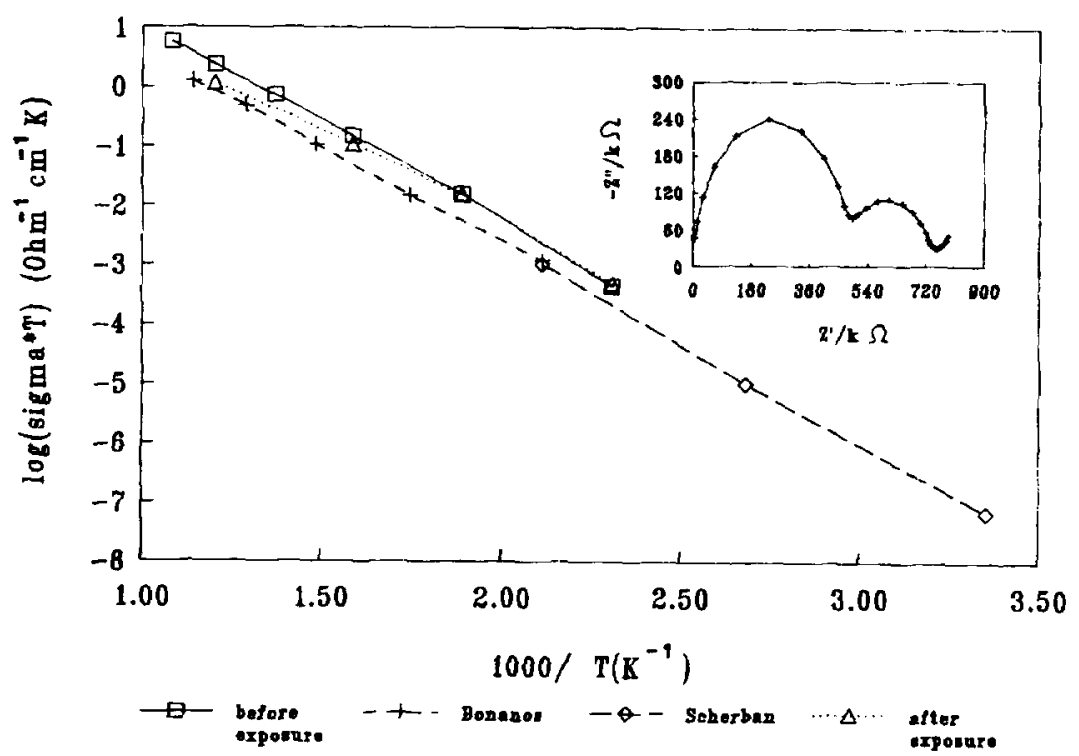

Fig. 2. Arrhenius plot of the temperature dependence of the bulk electrical conductivity for the $\mathrm{SrCe}_{0.95} \mathrm{Yb}_{0.05} \mathrm{O}_{3-\alpha}$ sample before and after exposure. The results are also compared with the results (refs. [3] and [4]) of other investigations. Insert: Impedance spectrum at $200^{\circ} \mathrm{C}$ for a specimen before exposure. 
al. $[1,2]$ indicate clearly that the preparation route needs further optimisation.

The present results provide a clear indication that the mechanical and electrical properties are practically not affected by an exposure to a simulated coal gas atmosphere.

An interpretation of the IS measurements can be made on the assumption that a dominant bulk conductivity prevails at the higher temperatures. In this case an Arrhenius curve of this bulk conductivity can be constructed and compared with the results of other investigations. As shown in fig. 2 , the electrical measurements yield nearly the same results before and after exposure to coal-gas atmosphere. Both data compare very well with the IS data of Scherban et al. [3] and Bonanos et al. [4] for bulk conductivity. The good correlation observed in this figure supports the assumption made. Also the activation energy value of $0.66 \mathrm{eV}$ is in good agreement with the reported values of $0.63 \mathrm{eV} \mathrm{[3]} \mathrm{and} 0.65 \mathrm{eV}$ [4], respectively. To conclude this discussion, it should be mentioned, that in order to prove protonic conduction in low $P_{\mathrm{O}_{2}}\left(10^{-20}\right)$ atmosphere, as occurring in a fluidised bed gasifier, IS experiments in these atmospheres are necessary.

\section{Conclusions}

In this study of strontium cerates for use in a hy- drogen sensor it is shown that the mechanical and electrical properties are not influenced substantially by exposure to a synthetic coal-gas atmosphere. However, the mechanical properties need to be improved by an optimization of the sintering procedure.

Impedance spectroscopy measurements reveal reliable bulk conductivity values. Data in the low $P_{\mathrm{O}_{2}}$ atmosphere are necessary in order to establish the protonic conduction in these circumstances.

\section{Acknowledgement}

The authors wish to thank P. Diels, W. Hendrix, J. Gedopt and their group for the careful characterization of the materials. They are also indebted to $P$. Lens and J. Cooymans for technical assistance. This work is sponsored by the CEC non-nuclear energy R\&D program, under JOULE contract number JOUF 0029-C.

\section{References}

[1] H. Iwahara, H. Uchida and S. Tanaka, Solid State lonics 9/ 10 (1983) 1021.

[2] H. Uchida, H. Yosshikawa and H. Iwahara, Solid State lonics 35 (1989) 229.

[3] T. Sherban and A.S. Nowick, Solid State Ionics 35 (1989) 189.

[4] Bonanos, B. Ellis and M.N. Mahmood, Solid State Ionics 2830 (1988) 579. 